\title{
Terminologie, Symbole, Abkürzungen
}

\section{Terminologie}

Die im Studienbuch verwendete Terminologie ist ohne Extravaganzen. Sie ist die der üblichen indogermanistischen Handbücher. In Einzelfällen wird a.O. dazu Genaueres gesagt, so bei den Termini für die Ablaut- und Flexionsmuster der Nomina (s.u. F 315 Abs. 3-4).

Eine zusätzliche Orientierung bietet das am Ende des Studienbuches beigefügte Sachregister. Es verweist auf die relevanten Paragraphen oder bringt weiterführende Literatur. Bleibt eine Frage offen, etwa im Bereich der hier nicht weiter behandelten Allgemeinen Sprachwissenschaft, dann stehen kompetente und gut informierende Lexika und Wörterbücher mit weiteren Hinweisen zur Verfügung: $\rightarrow$ Bussmann Lexikon d. Sprachw. 2. Aufl. 1990; Lewandowski Linguist. Wörterbuch 1-3 1994; Metzler Lexikon Sprache 2000. Bei speziellen terminologischen Einzelheiten in der idg. Lautlehre: $\rightarrow$ Sachregister bei Mayrhofer Lautlehre 1986 p. 182-185. Bei terminologischen Einzelheiten in der Formenlehre des uridg. Verbums: $\rightarrow$ Vorwort im LIV $1998=$ Lexikon der indogermanischen Verben 1998 p. 1ff. Bei Fragen bezüglich der Nominalbildung, des Wortschatzes und seiner Probleme: $\rightarrow$ Einführung in die Terminologie bei Kluge / Seebold 1995 p. XIVff. Sehr nützlich ist ferner das Register der Zeitschrift für Vergleichende Sprachforschung, s.u. in der Bibliographie s.v. HS = Historische Sprachforschung. Letzter Hinweis: $\rightarrow$ Duden Grammatik 1995 p. 828 ff. (Verzeichnis der Fachausdrücke).

Die Terminologie stammt im wesentlichen von den Griechen. Sprachtheoretische Erörterungen sind spätestens seit dem 5. Jh. v. Chr. nachzuweisen. Einen guten Einblick in den grammatischen Wissensstand der hellenistischen Zeit bietet Dionysios Thrax: $\rightarrow$ Dionysios Thrax Grammatik. Im Laufe des 2. Jh. v. Chr. werden die gr. Termini von den römischen Gelehrten übernommen und mehr oder weniger latinisiert: $\rightarrow$ Wakkernagel Vorlesungen I 1926 p. $13 \mathrm{ff}$. 
Die Benennungsmotive für die Termini sind für uns Moderne oft auf den ersten Blick nicht mehr einsichtig, vgl. unter vielen den t.t. gr. $\pi \tau \tilde{\omega} \sigma \varsigma$, , der als lat. cāsus und als dt. Fall bis heute gebräuchlich ist. Ausgangspunkt für diesen t.t. ist vermutlich die Vorstellung, daß sich jedes Nomen in der nominativischen Zitierform einem Stift vergleichbar in senkrechter, gerader Position (= casus rectus) befindet. Die Verwendungen als Akkusativ, als Genetiv usw. weichen von der geraden Lage ab, sind also ungerade (= casus obliquus) und können im verwendeten Bild mit verschieden tiefem Herabsinken $(\pi \tau \tilde{\omega} \sigma / \zeta)$ des Stiftes beschrieben werden: $\rightarrow$ Wackernagel Vorlesungen I 1926 p. 15. Statt eines Stiftes liegt nach einer anderen Tradition das Bild des Falls eines Würfels („Knöchels") zugrunde: $\rightarrow$ F. Murru in MSS 391980 p. 73ff. - Als

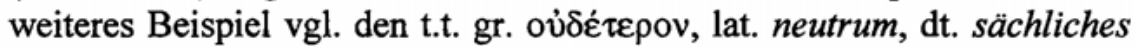
Geschlecht. Dahinter steckt nicht primär die Idee des „Weder-Noch“ ("keines der beiden Genera maskulin und feminin"), sondern vermutlich die Idee eines neben maskulin und feminin eigenständigen weiteren natürlichen „Mannweiber-genus": $\rightarrow$ K. Strunk Neutrum: zum antiken Benennungsmotiv eines grammatischen Terminus in FS Untermann Innsbruck 1993 p. $455 \mathrm{ff}$.

Die Geschichte der einzelnen sprachwissenschaftlichen Fachwörter und Fachausdrücke ist immer im Auge zu behalten. Alle neueren sprachwissenschaftlichen Ansätze fußen i.d.R. auf der sog. traditionellen Grammatik. Ihre erste Systematik hat sie in Auseinandersetzung mit dem klass. Latein erhalten. Die Anwendung auf andere Sprachen als Latein ist aber nicht unproblematisch: $\rightarrow$ Bussmann Lexikon d. Sprachw. 2. Aufl. 1990 p. 798f. Ferner darf man nicht übersehen, daß gerade in der modernen Allgemeinen Sprachwissenschaft ein und derselbe Terminus je nach Theorie verschieden verwendet werden kann, vgl. Lewandowski Linguist. Wörterbuch 31994 s.v. Terminus.

\section{Symbole, Schreibweisen, Zitierweisen}

\section{a. Symbole}

In der Regel sprechen die verwendeten Symbole und Zeichen für sich. Nicht von vornherein selbstverständlich sind die sog. Cover-Symbole:

$H=$ beliebiger Laryngal $\left(h_{1}, h_{2}\right.$ oder $h_{3}$ stehen dagegen für die eigentlichen Laryngale), s.u. L 314 
$K=$ beliebiger Konsonant $\left(d, d^{h}, t\right.$ usw. stehen dagegen für die eigentlichen Konsonanten)

$R=$ beliebiger Sonant ( $l, r$ usw. stehen dagegen für die eigentlichen Sonanten)

$V=$ beliebiger Vokal ( $e, o$ usw. stehen dagegen für die eigentlichen Vokale)

$\check{a} \quad=$ langer oder kurzer Vokal $a$ usw.

\# = Wortanfang oder Wortende

\#\# = Satzanfang oder Satzende

${ }^{*} m o n-u o-,{ }^{*} k^{w} i$ - usw. = - markiert die morphologische Segmentierung

*mon.uo-, "ra.i- usw. = . markiert die syllabische Segmentierung

$\emptyset$ oder $z \quad=$ Null(zéro)-Stufe, - Suffix, -Endung

$\circ=$ Schwachvokal, s.u. L 203

$\mathrm{W}=$ Wurzel

$\mathrm{S}=$ Suffix

$\mathrm{E}=$ Endung

$\mathrm{W}(e) \quad=-e$-Vollstufe der Wurzel

$\mathrm{W}(\bar{e}) \quad=-\bar{e}$-Dehnstufe der Wurzel

$\mathrm{W}(o) \quad=-o$-Vollstufe der Wurzel

$\mathrm{W}(\bar{o}) \quad=-\bar{o}$-Dehnstufe der Wurzel

$\mathrm{W}(\varnothing) \quad=$ Schwundstufe der Wurzel

$\mathrm{W}\left({ }^{\circ}\right) \quad=$ Reduktionsstufe d. Wurzel mit Schwachvokal, s.u. L 203

entsprechend:

$\mathrm{S}(e) \quad=-e$-Vollstufe des Suffixes, usw.

$\mathrm{E}(e) \quad=-e$-Vollstufe der Endung, usw.

* = nur rekonstruierte, nicht belegte Form (wenn bekannt, wird die vermutete Zeit der rekonstruierten Form mit urgr., uridg., voruridg. usw. angegeben)

$<=$ lautgesetzlich entstanden aus

$>\quad=$ entwickelt sich lautgesetzlich $\mathrm{zu}$

$=\quad=$ enstspricht, s.u. E 507 Abs. 5

$\Rightarrow=$ ersetzt durch

+ z.B. Hom. $+=$ Homer und später 
$\rightarrow$ der Pfeil verweist auf weiterführende Literatur außerhalb des Studienbuches (interne Querverweise werden mit s.o. oder s.u. angegeben)

lal die Schrägstriche verweisen auf den entsprechenden phonologischen Wert

[ $\eta$ ] die eckigen Klammern verweisen auf den entsprechenden phonetischen Wert

$<z>$ die spitzen Klammern markieren Grapheme (Schriftzeichen)

b. Schreibweisen

1) Die kürzlich eingeführte sog. neue deutsche Rechtschreibung wird nicht berücksichtigt.

2) Zur Notation des Urindogermanischen s.u. L $100 f$.

3) Bei den idg. Einzelsprachen werden die in den Handbüchern üblichen Schreibweisen verwendet. Auf ein paar Einzelheiten mache ich extra aufmerksam:

Im Lateinischen ist $\langle c\rangle$ immer als $/ k /$ zu verstehen, $\langle q u>$ dagegen als $/ k^{w} /$, ungeachtet dessen, ob es ein altes $/ k u /$ darstellt oder aus einem $/ k^{w} /$ entstanden ist, s.u. E $506 \mathrm{Abs}$. 3. Zu beachten ist ferner, daß ein Wort wie <maius> als/maiijus/ und ein <conicio> als /kon-iikiijo/ zu lesen ist: $\rightarrow$ Leumann LLFL 1977 p. 127f.

Bei den Beispielen aus dem mykenischen Gr. folgt in der Regel der Beleg zuerst so, wie ihn die myk. Linear-B-Schrift festhält (die Lautwerte der Silbenzeichen werden in lat. Schrift wiedergegeben). Danach wird die vermutliche phonologische Interpretation ebenfalls in lat. (nicht in gr. !) Schrift beigefügt (graphemisch mitbezeichnete phonetische Übergangslaute werden ebenfalls berücksichtigt, ferner erfolgt oft gleich eine moderne morphologische Segmentierung), vgl. (die Symbole $<>$ und / / werden der Einfachheit halber i.d.R. nicht geschrieben) $i$-je-ro-wo-ko i.e. hiiero-uorgos 'Priester'. - Wo eine Aspiration zu vermuten ist, wird sie durch $h$ markiert (ein Beispiel soeben). - Je nach der etymologischen Herkunft wird bei der Interpretation der $z$-Reihe ein $k^{j}$, ein $g^{j}$, ein $t^{j}$ oder ein $d^{j}$ eingesetzt, vgl. to-pe-za i.e. torped ${ }^{j} a$ 'Tisch' $<{ }^{*}\left(k^{w}\right)$ tr-ped-i $a$ 'Vierfüßler'. - Die Belegstellen der zitierten Formen sind nicht beigefügt, sie lassen sich aber leicht bei Aura Jorro DMic. I 1985 II 1993 ausfindig machen. - Beispiele aus gr. Dialektinschriften werden ohne Akzent geschrieben. 
Beim Altindischen (Vedischen) ist Mayrhofer EWAia Vorbild. Wie in den meisten sprachwissenschaftlichen Darstellungen üblich, werden hier die ved. und ai. Nominalformen i.d.R. als Stamm ohne Endung zitiert (also z.B. als ved. ávi- 'Schaf'). Ältere Handbücher setzen dafür gern den Nom.Sg. in der Sandhiform mit - $h$ (sog. Visarga), vgl. einen Verweis wie lat. ovis 'Schaf' = ved. ávih. Weiteres s.u. L 309 Abs. 3. - Bei ved. und ai. Verbalformen ist die 3.Pers.Sg. die Zitierform (vgl. ved. bhárati 'trägt'), z.T. auch die vollstufige Verbalwurzel (vgl. ved. bhar- 'tragen'). Akzente werden bei finiten Verbalformen nur gesetzt, wenn sie textlich belegbar sind.

Beim Avestischen folge ich $\rightarrow$ Hoffmann / Forssman Avestische Lautund Flexionslehre 1996.

Beim Anatolischen (Hethitischen) sollte $h$ sowohl bei der Umschrift aus der Keilschrift als auch beim Interpretament geschrieben werden, beim $\check{s}$ nur in der Umschrift, nicht aber beim Interpretament, vgl. z.B. pa$a h-h u$-e-na-aš i.e. pahhuenas. Das in der Umschrift eingebürgerte $z$ wird als $t^{S}$ wiedergegeben. Ferner, angeblich betonte Silbenzeichen wie $t a ́ k$ in ták-na-a-aš weisen damit nicht auf den Sitz des Akzents. Mit dem markierten $a ́$ wird nach den Gepflogenheiten der Altorientalistik lediglich angedeutet, daß neben der üblichen Form $\operatorname{tak}$ ( $=\operatorname{tak} \mathrm{Nr}$. eins) eine zweite Zeichenform ( $=$ tak Nr. zwei) in Verwendung ist.

Zum Gotischen: $\rightarrow$ Binnig Gotisch 1999.

\section{c. Zitierpraxis}

Es ist zu beachten, daß die Zitierpraxis nicht bei allen altidg. Sprachen gleich gehandhabt wird, vgl. bei den Verbalformen das lat. und das gr. System, wo die 1.Sg. Nennform ist; im Ved. aber wird traditionell die 3.Sg. zitiert, s.o. Abs. b.

Bei Mayrhofer KEWA werden die Verbalformen unter der 3.Sg. aufgelistet, bei Mayrhofer EWAia stehen sie aber unter der vollstufigen Verbalwurzel verzeichnet, vgl. zu ersterem p. III 562f. den Eintrag svárati 'gibt einen Ton von sich, tönt, erschallt, singt, besingt', zu letzterem II p. 792f. den entsprechenden Eintrag unter SVAR 'einen Ton von sich geben, tönen, schnauben, erschallen, singen'. Auch bei den Nomina ändert sich die Zitierweise des EWAia gegenüber der des KEWA, vgl. im KEWA svargáh, dagegen im EWAia svargá-. 


\section{Abkürzungen}

Wo allein der Ausgang -isch gekürzt ist, wird auf die Auflistung der Abkürzung verzichtet. In der Regel sind die Abkürzungen längst eingebürgert oder von sich aus im entsprechenden Kontext verständlich.

N.B.: Abkürzungen von Zeitschriften finden sich in der Bibliographie.

a.a.O. am angegebenen Ort

aav. altavestisch

Abl. Ablativ

Abs. Absatz

Abstr. Abstraktum

Adj. Adjektiv

Adv. Adverb

aengl. altenglisch

aheth. althethitisch

ahd. althochdeutsch

ai. altindisch

air. altirisch

Akk. Akkusativ

aksl. altkirchenslavisch

akt. aktivisch

Akt. Aktiv

alb. albanisch

an. altnordisch

Anm. Anmerkung

a.O. am Ort

Aor. Aorist

Aor.St. Aoristst.

ap. altpersisch

ark. arkadisch

arm. armenisch

As. Altsächsisch

ass. assimiliert

Ass. Assimiliation

athem. athematisch av. avestisch

AV Atharvaveda

Bd. Band

bzw. beziehungsweise

c. (Genus) commune

D Dehnstufe

Dat. Dativ

ders. denselben

Desid. Desiderativ

d.h. das heißt

dial. dialektal

Diath. Diathese

dies. dieselbe

diss. dissimiliert

Diss. Dissimilation

dt. deutsch

Du. Dual

dur. durativ

E Endung

enkl. enklitisch

ev. eventuell

f. (genus) femininum

ff. und folgende

FS Festschrift 
Fut. Futurum

FW Fremdwort

Gen. Genetiv

germ. germanisch

GN Göttername

gr. griechisch

gramm. grammat.

GS Gedenkschrift

GW Gegenwart

$H \quad$ Laryngal, ferner s.u. L 314

hell. hellenistisch

Hes. Hesiod

heth. hethitisch

HG Hinterglied

hom. homerisch

Hom. Homer

hrsg. herausgegeben

HS Hauptsatz

id. idem

idg. indogermanisch

i.d.R. in der Regel

iir. indoiranisch

Iir. Indoiranisch

Ind. Indikativ

Inf. Infinitiv

Inj. Injunktiv

inkl. inklusiv

Instr. Instrumental

intr. intransitiv

Ipf. Imperfekt

Ipt. Imperativ

i.S.v. im Sinn von

jav. jungavestisch

Jh. Jahrhundert

jheth. junghethitisch

Jt. Jahrtausend
$K \quad$ beliebiger Konsonant

Kaus. Kausativ

kluw. keilschrift-luwisch

KN Knossos

Koll. Kollektiv

Konj. Konjunktiv

kons. konsonantisch

Kons. Konsonant

KP Kompositum

KS Kleine Schriften

lat. lateinisch

lit. litauisch

Lit. Literatur

Lok. Lokativ

lt. laut

LW Lehnwort

m. (genus) masculinum

m.E. meines Erachtens

med. medial

Med. Medium

mhd. mittelhochdeutsch

mir. mittelirisch

mp. mittelpersisch

MY Mykene

myk. mykenisch

n. (genus) neutrum

nb. neben

N.B. nota bene!

N.F. Neue Folge

nhd. neuhochdeutsch

Nom. Nominativ

Nom.act. Nomen actionis

Nom.ag. Nomen agentis

NS Nebensatz

N.S. Neue Serie

Ntr. Neutrum

Num. Numerus 

o.ä. oder ähnlich
ON Ortsname
Opt. Optativ

p. Seite (pagina)

P. Person

pal. palaisch

Pass. Passiv

Perf. Perfekt

Perf.St. Perfektstamm

Pers. Person

PK Possessivkompositum

Pl. Plural

Plt. Plautus

Plpf. Plusquamperfekt

PN Personenname

poss. possessiv

PPA Partizip Perfekt Aktiv

PPP Partizip Perfekt Passiv

Präs. Präsens

Präs.St. Präsensstamm

prim. primär

Pron. Pronomen

Ptz. Partizip

PY Pylos

$R \quad$ beliebiger Sonant

redupl. redupliziert

Redupl. Reduplikation

refl. reflexiv

Refl.Pron. Reflexivpronomen

rel. relativ

Rez. Rezension

RS Relativsatz

RV Rgveda

s. siehe

S Suffix

SA Satzanfang

sc. scilicet schw. schwach

SE Satzende

sek. sekundär

Sek. Sekundär-

sem. semitisch

Sg. Singular

s.o. siehe oben

sog. sogenannt

Sp. Spalte

st.St. starker Stamm

St. Stamm

sth. stimmhaft

stl. stimmlos

s.u. siehe unten

Subst. Substantiv

südgr. südgriechisch

Suff. Suffix

s.v. sub voce

TB Taittiriya Brahmana

TH Theben

them. thematisch

thess. thessalisch

toch. tocharisch

tr. transitiv

t.t. Terminus technicus

u.a. und andere(s)

u.a.m. und andere(s) mehr

u.a.O. und andere Orte

urgerm. urgermanisch

urgr. urgriechisch

uridg. urindogermanisch

usw. und so weiter

$\begin{array}{ll}V & \text { beliebiger Vokal } \\ \text { V } & \text { Vollstufe } \\ \text { VA } & \text { Versanfang } \\ \text { VE } & \text { Versende } \\ \text { VG } & \text { Vergangenheit }\end{array}$


VG (bei KP) Vorderglied

vgl. vergleiche

VN Völkername

vs. versus

WA Wortanfang

WE Wortende

Wn. Wurzelnomen
Wz. Wurzel

Wz.Aor. Wurzelaorist

Wz.Präs. Wurzelpräsens

z.B. zum Beispiel

z.T. zum Teil

z.Z. zur Zeit 